\title{
OBSERVATÓRIO DA ENFERMAGEM: FERRAMENTA DE MONITORAMENTO DA COVID-19 EM PROFISSIONAIS DE ENFERMAGEM
}

Marcelo Felipe Moreira Persegona ${ }^{1}$ Ricardo Antônio Ribeiro Pires ${ }^{2}$ Gilney Guerra de Medeiros ${ }^{2}$ Flaviana Alves dos Santos Pinheiro² Miriam Santos da Silva Lopes ${ }^{2}$ Antonio Nascimento Junior ${ }^{2}$ Manoel Carlos Neri da Silva ${ }^{1}$ David Lopes Neto

\author{
https://orcid.org/0000-0002-8430-9317 \\ https://orcid.org/0000-0003-1953-2034 \\ https://orcid.org/0000-0002-3351-2841 \\ https://orcid.org/0000-0001-6383-8423 \\ https://orcid.org/0000-0002-4999-7265 \\ https://orcid.org/0000-0002-8303-7487 \\ https://orcid.org/0000-0002-3923-7473 \\ https://orcid.org/0000-0002-0677-0853
}

Objetivo: Apresentar o Observatório da Enfermagem como um veículo oficial de comunicação do Sistema Conselho Federal de Enfermagem/ Conselhos Regionais de Enfermagem, sobre a situação epidemiológica da COVID-19 na Enfermagem brasileira. Método: Estudo sobre o desenvolvimento de um sistema eletrônico, do tipo observatório, construido pelo Departamento de Tecnologia de Informação e Comunicação, do Conselho Federal de Enfermagem, com o intuito de acompanhar a epidemiologia da COVID-19 entre os profissionais de Enfermagem. Resultados: O Observatório da Enfermagem é um sistema que possui um formulário eletrônico estruturado, o qual permite a coleta e análise de dados sobre a propagação da COVID-19 nos profissionais de Enfermagem em todo o território nacional. A criação, implantação e implementação do Observatório da Enfermagem e suas quatro etapas deram origem ao painel de indicadores com a notificação de casos de infecção e óbitos de profissionais de Enfermagem pela COVID-19. Considerações Finais: O Observatório da Enfermagem como sistema de informação sobre a incidência de casos e a ocorrência de óbitos por COVID-19 tem se mostrado uma importante ferramenta gerencial para tomada de decisão em todo o território nacional, seja pelas entidades de Enfermagem ou pelos Gestores do Sistema Único de Saúde nas três esferas de governo e da inciativa privada; bem como tem contribuido para dá maior visibilidade ao trabalho dos profissionais de Enfermagem e desvelar as precárias condições de trabalho a que estes estão expostos, junto à população e a imprensa.

Descritores: Enfermagem; Profissionais de Enfermagem; Pandemia; Infecções por coronavírus; COVID-19.

\section{NURSING OBSERVATORY: COVID-19 MONITORING TOOL IN NURSING PROFESSIONALS}

Objective: To present the Nursing Observatory as an official communication vehicle of the Federal Nursing Council/Regional Nursing Councils system, on the epidemiological situation of COVID-19 in Brazilian Nursing. Method: Study on the development of an observatory-type electronic system, built by the Department of Information and Communication Technology, of the Federal Nursing Council, in order to monitor the epidemiology of COVID-19 among nursing professionals. Results: The Nursing Observatory is a system that has a structured electronic form, which allows the collection and analysis of data on the spread of COVID-19 among nursing professionals across the national territory. The creation, implementation and implementation of the Nursing Observatory and its four stages gave rise to the panel of indicators with the notification of cases of infection and deaths of nursing professionals by COVID-19. Final Considerations: The Nursing Observatory as an information system on the incidence of cases and the occurrence of deaths by COVID-19 has been shown to be an important managerial tool for decision making throughout the national territory, whether by Nursing entities or Managers the Unified Health System in the three spheres of government and the private initiative; as well as it has contributed to give greater visibility to the work of Nursing professionals and to reveal the precarious working conditions to which they are exposed, together with the population and the press.

Descriptors: Nurse; Nurse Practitioners; Pandemic; Coronavirus Infections; COVID-19.

\section{OBSERVATORIO DE ENFERMERÍA: HERRAMIENTA DE MONITOREO COVID-19 EN PROFESIONALES DE ENFERMERÍA}

Objetivo: Presentar el Observatorio de Enfermería como vehículo de comunicación oficial del sistema del Consejo Federal de Enfermería / Consejos Regionales de Enfermería, sobre la situación epidemiológica de COVID-19 en la Enfermería brasileña. Método: Estudio sobre el desarrollo de un sistema electrónico de tipo observatorio, construido por el Departamento de Tecnología de la Información y la Comunicación, del Consejo Federal de Enfermería, para monitorear la epidemiología de COVID-19 entre los profesionales de enfermería. Resultados: El Observatorio de Enfermería es un sistema que tiene una forma electrónica estructurada, que permite la recopilación y el análisis de datos sobre la propagación de COVID-19 entre los profesionales de enfermería en todo el territorio nacional. La creación, implementación e implementación del Observatorio de Enfermería y sus cuatro etapas dieron lugar al panel de indicadores con la notificación de casos de infección y muerte de profesionales de enfermería por COVID-19. Consideraciones finales: El Observatorio de Enfermería como un sistema de información sobre la incidencia de casos y la ocurrencia de muertes por COVID-19 ha demostrado ser una importante herramienta de gestión para la toma de decisiones en todo el territorio nacional, ya sea por entidades de enfermería o gerentes el Sistema Único de Salud en las tres esferas del gobierno y la iniciativa privada; así como ha contribuido a dar mayor visibilidad al trabajo de los profesionales de enfermería y a revelar las precarias condiciones de trabajo a las que están expuestos, junto con la población y la prensa.

Descriptores: Enfermería; Enfermeras Practicantes; Pandemía; Infecciones por Coronavirus; COVID-19.

${ }^{1}$ Conselho Federal de Enfermagem (Cofen). Brasilia, DF.

'Universidade de Brasilia (UNB). Faculdade de Economia, Administração, Contabilidade e Gestão de Políticas Públicas (FACE). Brasilia, DF.

${ }^{3}$ Universidade Federal do Amazonas (UFAM). Escola de Enfermagem de Manaus. Manaus, AM.

Autor Correspondente: Marcelo Felipe Moreira Persegona. E-mail: marcelo.persegona@gmail.com

Recebido: 07/7/20 - Aceito: 09/8/20 


\section{INTRODUÇÃO}

Observatórios profissionais são essenciais às instituições ou entidades que buscam monitorar e avaliar situações de trabalho e saúde de trabalhadores ao longo do tempo ${ }^{1}$. A palavra observatório tem sua origem no campo da astronomia, significando "local onde são feitas observações, onde se observa", sendo os primeiros os relacionados a astronomia e meteorologia, como como os observatórios de Mauna Kea, Very Large Array, Observatório Real de Greenwich, Observatório Paranal, Observatório Astronômico Sul-Africano².

Outro exemplo de observatório, o Social Watch, Observatório da Cidadania em português, Control Ciudadano em espanhol, é uma coalizão de 105 entidades da sociedade civil de 50 paises de todas as regiões do mundo que atuam na fiscalização sobre as ações governamentais em prol da melhoria das condições de vida das populações, monitorando as políticas públicas em âmbito global ${ }^{3}$.

Com os adventos da globalização e da era digital, observatórios de diversas naturezas foram criados com o intuito de dar visibilidade, transparência, divulgação de informações e dados fidedignos de instituições e empresas por meio da internet. Nesse sentido, em meio à chegada da pandemia de COVID-19 no Brasil, em meados de março de 2020, o Conselho Federal de Enfermagem (Cofen), constituiu o Comitê Gestor de Crises $^{4}$ (CGC-Cofen), ao qual coube a criação do "Observatório da Enfermagem"5 disponibilizado em seu site, com a finalidade de gerar informações sobre o avanço da COVID-19 e a situação de saúde dos profissionais de Enfer- magem no Brasil, com valor agregado para os tomadores de decisão do Sistema Cofen/Conselhos Regionais de Enfermagem.

O estudo tem por objetivo apresentar o Observatório da Enfermagem como um veículo oficial de comunicação do Sistema Cofen/Conselhos Regionais de Enfermagem, sobre a situação epidemiológica da COVID-19 na Enfermagem brasileira.

\section{DESENVOLVIMENTO}

Este estudo, de descrição de inovação tecnológica, foi desenvolvido e operacionalizado pelo Departamento de Tecnologia de Informação e Comunicação (DTIC), do Cofen a partir março de 2020, como demanda estratégica em resposta a situação pandêmica que afetou enormemente os profissionais de Enfermagem.

O Observatório da Enfermagem (Figura 1) é um sistema de tecnologia da informação e comunicação que possui um formulário eletrônico estruturado, o qual permite a coleta, identificação e análise de dados sobre a propagação da COVID-19 e seu acometimento nos profissionais de Enfermagem em todo o território nacional. Configurando-se em um ambiente computacional em tempo real de análise de dados e uma ferramenta de apoio à tomada de decisão, a qual facilita o reconhecimento, processamento e acesso à informação e, posterior divulgação para organismos brasileiros federais, estaduais e municipais, organizações internacionais, sociedade brasileira e veículos de imprensa nacional e internacional.

Figura 1 - Sítio de Internet do Observatório da Enfermagem, Cofen.
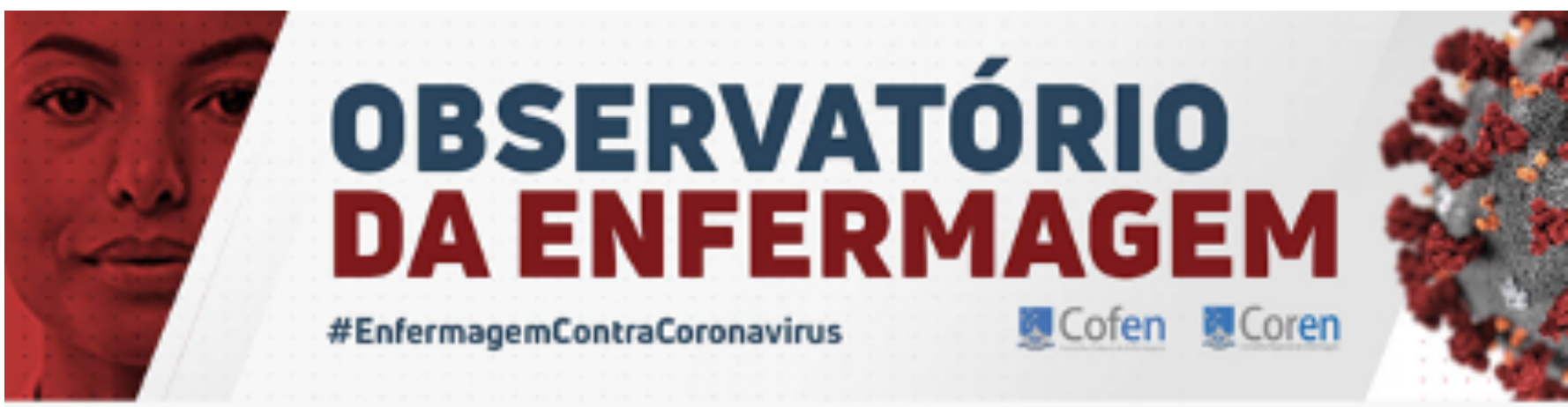

comumiqur movos casos

ot Conthaminaclo pos

CORONEVIRUS (COVID-19) 
Figura 1 - Sítio de Internet do Observatório da Enfermagem, Cofen.

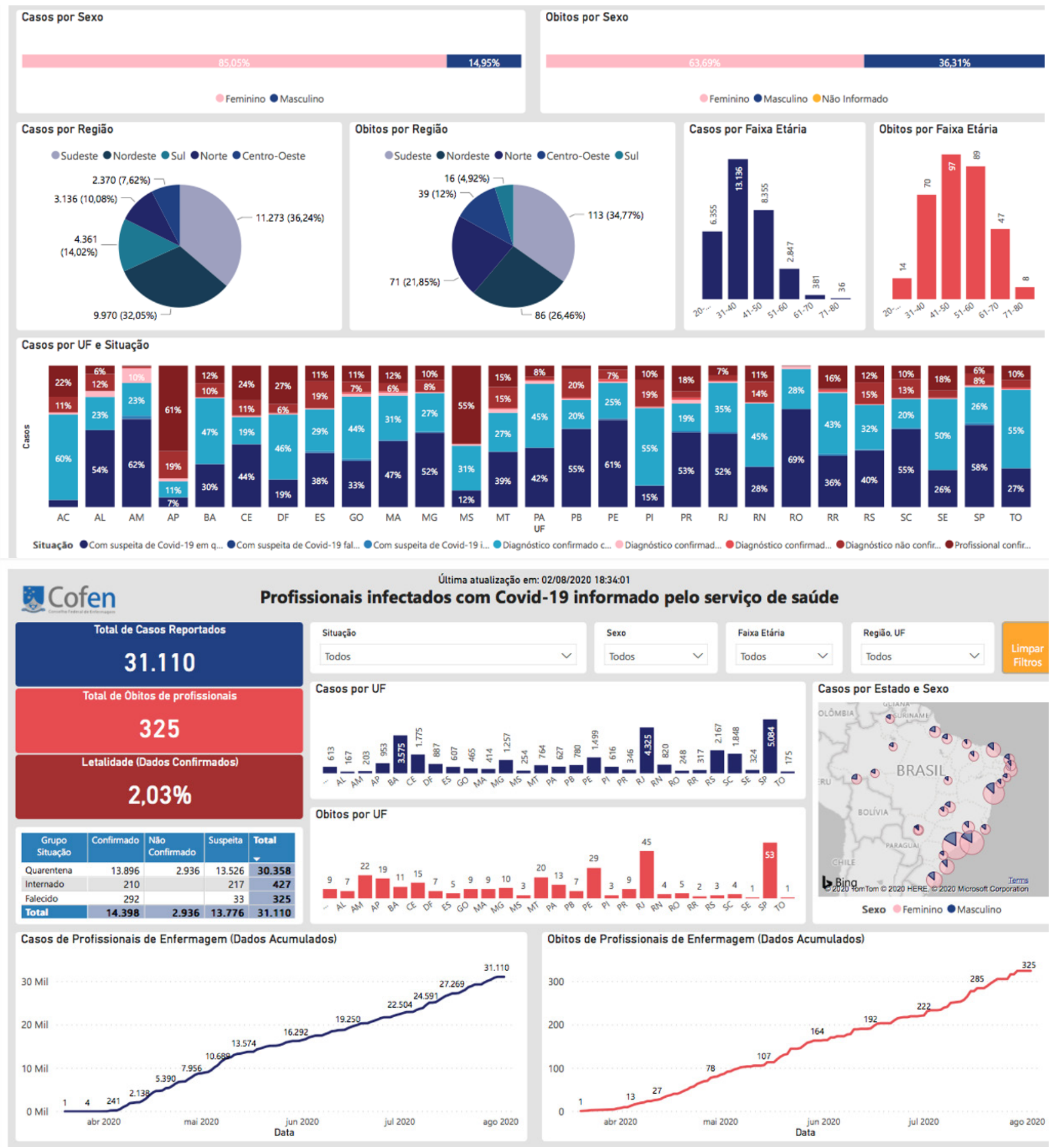

Para sua construção, o Observatório de Enfermagem passou por quatro etapas:

Etapa 1 - Formulário Eletrônico: criação de formulário online no Google Docs, com a análise dos dados por meio de planilha eletrônica do Excel e, que em seguida são disponibilizados em uma página de internet criada a partir do Google Maps pelo Departamento de Tecnologia de Informação e Comunicação (DTIC/Cofen). Essa página é alimentada pela equipe do Observa- 
tório da Enfermagem.

Etapa 2 - Painel de Indicadores: desenvolvido para fornecer visualizações interativas e com uma interface simples para criação de relatórios e dashboards a partir dos dados do banco criado no Excel. O painel de indicadores deu origem ao site do Observatório da Enfermagem (http://observatoriodaenfermagem.cofen. gov.br/)

O Observatório da Enfermagem notifica a ocorrência de casos de infecção e óbitos dos profissionais de Enfermagem pela COVID-19. Os casos para integrarem o observatório, passam pela seguinte análise: se é suspeita de COVID-19, em quarentena; se possui diagnóstico confirmado com COVID-19, em quarentena; diagnóstico não confirmado de COVID-19; com suspeita de COVID-19 e internado; diagnóstico confirmado com COVID-19 e internado; e profissional confirmado com COVID-19 e com alta.

Os casos de óbitos possuem as seguintes situações: falecidos com suspeita de COVID-19 e falecidos com diagnóstico confirmado de COVID-19. Os dados recebidos passam pelo processo de Extração, Tratamento e Limpeza (ETL) antes de serem disponibilizados para elaboração dos relatórios e atualização da base de dados do Observatório da Enfermagem. Este processo está estruturado na análise por Unidade da Federação (UF) e macrorregião onde ocorreu o caso, categoria profissional do infectado, idade, classificação da faixa etária. Em seguida, ocorre a produção da lista, com a quantidade de casos de infecção e óbitos de profissionais de Enfermagem por UF para confecção dos cartogramas.

Etapa 3 - Cadastramento de Dados Profissionais: etapa na qual os dados podem ser inseridos na plataforma do Observatório de Enfermagem pelos profissionais de Enfermagem infectados pela COVID-19. Este formulário eletrônico reduz a quantidade de dados necessários para serem enviados e realiza validação com o banco de dados do S-GEnf do Cofen que alimenta o painel de indicadores em tempo real. Ressalta-se que nessa etapa ocorreu a substituição dos formulários do Google Docs pelo formulário eletrônico único do próprio sistema, em 12 de maio de 2020, Dia Internacional da Enfermagem e Dia do Enfermeiro 5 .

Nesse novo formulário eletrônico são aplicadas regras de verificação dos dados enviados, como por exemplo: categoria profissional e número de registro profissional, esta dupla de dados é checada no Sistema de Gestão da Enfermagem (S-GEnf). Detectados suas existências no sistema, são preenchidos outros dados necessários para análise, como: idade e sexo do profissional de Enfermagem, caso contrário, o sistema do observatório apresenta um novo formulário, no qual são enviados os dados dos profissionais não localizados no S-GEnf. Esta nova sistemática simplificou o recebimento dos dados e reduziu, consideravelmente, o seu tratamento, bem como, a elaboração de relatórios de três horas para 45 minutos, aumentando a eficiência do serviço.

Etapa 4 - Tratamento dos dados: nessa etapa a equipe da Assessoria de Comunicação passa a receber e tratar dados de óbitos de profissionais de Enfermagem no Sistema do Observatório da Enfermagem. Este processo reduz o tempo de processamento e checagem de dados de óbitos de profissionais e dá consistência entre os dados da Assessoria de Comunicação e do Observatório da Enfermagem.

O sítio de internet do Observatório da Enfermagem é o depositório do processo de tratamento e disponibilização da informação do Observatório da Enfermagem envolve cinco principais atores, os profissionais de Enfermagem, Coordenadores/Representantes Técnicos e Comissão de Gestão de Crises dos Conselhos Regionais de Enfermagem; Ministérios da Saúde, do qual são extraídos os dados de infecção e óbitos da população brasileira; Assessoria de Comunicação do Cofen que fornece as informações válidas de óbitos dos profissionais de Enfermagem e DTIC/Cofen que atualiza os dados da base de dados do Observatório da Enfermagem após a Assessoria de Planejamento e Gestão ter validados os dados recebidos e gerados os relatórios de controle e acompanhamento da infecção dos profissionais de Enfermagem. O processo de tratamento de dados de infectados e óbitos pela COVID-19 é realizado todos os dias, inclusive finais de semana e feriados.

As atualizações dos dados sobre casos de infecção e óbitos suspeitos e confirmados por COVID-19 de profissionais de Enfermagem no Brasil é realizado diariamente pelo Comitê Gestor de Crises do Cofen por meio dos dados enviados por profissionais, Coordenadores/ Representantes Técnicos e Comissões de Gestão de Crises dos Conselhos Regionais de Enfermagem.

Os dados fornecidos são consolidados e disponibilizados publicamente todos os dias, de seis em seis 
horas no site do Cofen e uma vez por dia é emitido o relatório estático para os tomadores de decisão do Sistema Cofen/Conselhos Regionais de Enfermagem. O processo de atualização das informações recebidas é dinâmico e complexo. Os dados informados diariamente estão sujeitos a verificação, o que pode provocar mudanças no número de casos ou óbitos em decorrência da detecção de erros ou atrasos no repasse das informações. Os casos e óbitos são atualizados por data e fonte de notificação.

Os óbitos dos profissionais de Enfermagem só são aceitos após passar por análise da Assessoria de Comunicação do Cofen que realiza contato com o hospital, familiar ou local onde ocorreu o óbito para verificar a veracidade do informe e depois atualiza a planilha eletrônica de óbitos, haja vista que começaram a serem fornecidas informações não verídicas (fake news) por parte do público externo. Com isso, as informações passaram a ser checadas a partir de fontes fidedignas e, somente após a confirmação, são descartadas ou inseridas na plataforma digital.

Ressalta-se que um observatório eletrônico de Saúde e de Enfermagem é uma fonte autorizada e respeitada de informações que apoiam tomadas de decisões e o desenvolvimento de políticas e aumentam o conhecimento sobre determinado tema ou assunto dos profissionais ${ }^{1}$. Ainda mais nesse momento de pandemia, em que na linha de frente estão os trabalhadores de Enfermagem, que somam mais de 2,3 milhões, em todo o país; e que a depender da dimensão desta milhares de profissionais poderão se contaminar ou ate mesmo morrer, em decorrência do trabalho ${ }^{7}$, sendo o Observatório de Enfermagem uma importante ferramenta de gestão e de análise situacional dos trabalhadores da Enfermagem

A consolidação das informações recebidas das fontes deu origem a uma base de dados elaborada em planilha eletrônica que produz dois relatórios, um em forma de tabelas e outro em forma de gráficos. Os dados do Observatório, agregados por estado, faixa etária, gênero e situação da doença (quarentena, internamento ou óbito) traçam um panorama da Enfermagem no contexto da pandemia no Brasil.

Nessa linha de construção de observatório no campo da saúde, estudo sobre uma proposta de um observatório epidemiológico do Sistema Único de Saúde (SUS) apontou que o fornecimento de dados em saúde é fundamental para a melhoria da qualidade de sistemas de saúde e servem de guias para investigações e tomada de decisão provenientes de relatórios informativos ${ }^{8}$.

\section{Limitações Da inovação}

Trata-se de um produto tecnológico digital em fase de implantação para gerenciamento de dados sobre incidência casos e mortalidade por COVID-19 em profissionais de Enfermagem em todo Brasil, o que requer a alimentação diária do sistema pelos Conselhos Regionais de Enfermagem, não tendo o DTIC-Cofen gerência sobre a inserção das informações, podendo ocorrer informações incompletas e subnotificação.

\section{Contribuições para a prática}

O Observatório de Enfermagem é uma ferramenta gerencial que facilitará a análise epidemiológica da situação de saúde dos trabalhadores de Enfermagem nos serviços de saúde com o fornecimento em tempo real de dados aos gestores dos conselhos regionais de enfermagem para tomada de decisão dentro das atividades finalísticas dessas autarquias.

\section{CONSIDERAÇÕES FINAIS}

A iniciativa mostra evidências de que a criação do Observatório da Enfermagem representa uma valiosa colaboração do Cofen para o monitoramento e o acompanhamento da COVID-19 nos profissionais de Enfermagem e contribui para a redução de custos para o Sistema Cofen/Conselhos Regionais de Enfermagem, como ferramenta de comunicação perante a mídia nacional e internacional, agências de saúde nacionais e internacionais, profissionais de enfermagem e a sociedade brasileira e tomada de decisão gerencial.

O Sistema Cofen/Conselhos Regionais de Enfermagem alcançou diversos resultados positivos com o Observatório da Enfermagem, ampliando a interação entre o próprio sistema entre si e com os stakeholders do Observatório da Enfermagem, além de melhoria da imagem corporativa da autarquia e, por conseguinte, dos profissionais de Enfermagem nas agências de saúde governamentais nacionais e internacionais, poder público brasileiro e imprensa, com maior valorização da profissão e visibilidade do Sistema Cofen/Conselhos Regionais de Enfermagem no enfrentamento da COVID-19.

Contribuição dos Autores: Os autores participaram de todas as etapas do estudo: desenho do estudo; coleta das informações no sistema; redação do manuscrito e aprovação da versão final revisada. 


\section{REFERÊNCIAS}

1. Bartz CC, Hardiker NR, Coenen A. Toward a Global eHealth Observatory for Nursing. Stud Health Technol Inform. [internet] [cited 2020 Jun 12]. 216:1114.-1114. Available from: https:// europepmc.org/article/med/26262413.

2. Bhattacharya B. Do you love to look at the stars? Here are 5 of the best places to do so. October 18, 2016. Outlook Traveller. [Internet] 2016 Out 16 [cited 2020 Jun 12]. Available from: https://www.outlookindia.com/outlooktraveller/ explore/story/25970/5-best-observatories-inthe-world.

3. Aguiar S. Observatório da cidadania: monitorando as políticas públicas em âmbito global. Ci. Inf., [internet] 1999 [cited 2020 Jun 12], 28(2):139-145. Available from:<http://www.scielo. br/scielo.php?script=sci_arttext\&pid=S010019651999000200006\&lng=en\&nrm=iso>. Access on 02 Aug. 2020. https://doi.org/10.1590/ S0100-19651999000200006.

4. Santos V, Persegona M, de Souza E, Almeida W, Filete M, da Silva, M.Comitê gestor de crise do coronavírus no âmbito do Cofen. Enferm Foco, [internet] 2020 [cited 2020 Jun 12]; 11(2):6-10. Available from <http://revista.cofen.gov.br/ index.php/enfermagem/article/view/4213>. Acesso em: 02 ago. 2020. doi: https://doi. org/10.21675/2357-707X.2020.vll.n2.4213

5. Conselho Federal de Enfermagem (COFEN).
Observatório de Enfermagem. Profissionais infectados com COVID-19 informado pelos enfermeiros responsáveis técnicos/ coordenadores. [Internet] 2020 [cited 2020 May 18]. Available from: http:// observatoriodaenfermagem.cofen.gov.br/

6. Silva MTN. Semana Brasileira de Enfermagem. Rev. bras. enferm. [Internet] 1997 June [cited 2020 Jun 11]; 50(2):151152. Available from: http://www.scielo.br/ scielo.php?script=sci_arttext\&pid=S003471671997000200001\&lng=en.

https://doi. org/10.1590/S0034-71671997000200001. Lourenção LG. A Covid-19 e os desafios para o sistema e os profissionais de saúde. Enferm Foco [internet] 2020 [cited 2020 Jun 11]; 11(1):67. Available from: <http://revista.cofen.gov.br/ index.php/enfermagem/article/view/3488> doi:https://doi.org/10.21675/2357-707X.2020 v1l.n1.3488

7. Saldanha RF, Bastos RR, BustamanteTeixeira MT, Leite Isabel Cristina Gonçalves, Campos Estela Márcia Saraiva. Proposta de um observatório epidemiológico do Sistema Único de Saúde. Cad. Saúde Pública [Internet] 2017 [cited 2020 Aug 02]; 33(1):e00113216. Available from: http://www.scielo.br/ scielo.php?script=sci_arttext\&pid=S0102311X20170001060018lng=en. Epub Jan 23 2017 https://doi.org/10.1590/0102-

$311 \times 00113216$ 